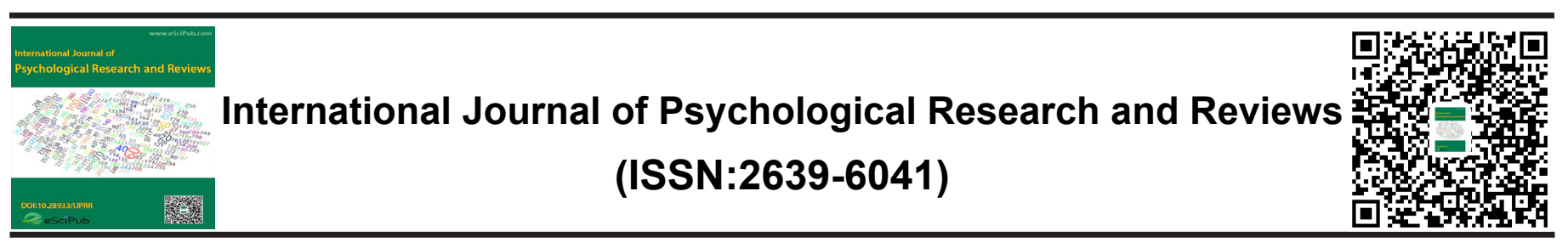

\title{
Social Media, Physical and Mental Health During a COVID-19 Lockdown
}

\author{
Tiffany Field ${ }^{1,2}$, Samantha Poling ${ }^{2}$, Shantay Mines ${ }^{2}$, Debra Bendell ${ }^{2}$, Connie Veazey ${ }^{2}$ \\ ${ }^{1}$ University of Miami/Miller School of Medicine \\ ${ }^{2}$ Fielding Graduate University
}

\begin{abstract}
Social media including texting, internet use, and Facebook time have differential effects but those have not been studied during the social isolation of lockdowns when they might be more prevalent. In this Survey Monkey study, as many as $98 \%$ of 260 respondents reported texting, 100\% using the internet, and $91 \%$ being on Facebook. The percentiles for those using the different media "a lot" were $45 \%, 77 \%$ and $42 \%$ respectively. Correlation analyses suggested that texting and internet use were positively related to Connecting Scale scores. However, internet use was also positively related to scores on Stress, Anxiety and Depression Scales and Facebook use was positively related to not only scores on Stress, Anxiety and Depression Scales but also to scores on Fatigue, Sleep Disturbance and PTSD scales. ANOVAS based on comparisons between groups reporting no to moderate use versus "a lot" of use were confirmatory of the correlation analyses. These results are limited by their being self-reported data from a non-representative, cross-sectional sample. Nonetheless, they highlight the positive and negative effects of different social media during a COVID-19 lockdown.
\end{abstract}

*Correspondence to Author:

TiffanyField ${ }^{1,2}$

${ }^{1}$ University of Miami/Miller School of Medicine; ${ }^{2}$ Fielding Graduate University

How to cite this article:

Tiffany Field, Samantha Poling, Shantay Mines, Debra Bendell, Connie Veazey. Social Media, Physical and Mental Health During a COVID-19 Lockdown. International Journal of Psychological Research and Reviews, 2021, 4:48

Keywords: Social Media; Physical and Mental Health; COVID-19

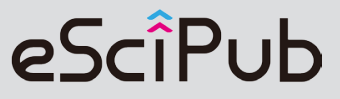
eSciPub LLC, Houston, TX USA.

Website: https://escipub.com/ Lockdown 
Social media has had mixed effects on physical and mental health. Mobile apps and messaging have been used to improve physical health and decrease anxiety, stress and depression. ${ }^{1}$ And, web-based interventions were reputedly as effective as human interventions in at least one study. ${ }^{2}$ However, social media in natural settings has had negative effects especially when excessively used.

Texting has been related to greater anxiety. ${ }^{3}$ And, in another recent study and review, it was related to both greater anxiety and depression. ${ }^{4,5}$. Sleep disturbances have also been associated with excessive texting. In a recent review of the literature on texting, excessive use was associated with shorter sleep and inferior sleep quality. ${ }^{6}$ And, texting in bed has especially contributed to both anxiety and sleep disturbances including insomnia and shorter sleep duration. ${ }^{7}$ As in most crosssectional studies, the direction of effects cannot be determined. Without baseline data and longitudinal research, it is unknown whether texting leads to anxiety, depression and sleep disturbances or vice versa, with texting being a coping mechanism.

Similarly, much of the recent research on internet use has suggested negative effects. Excessive Internet use has been associated with depression. ${ }^{8}$ And, Internet addiction has been related to both depression and anxiety. ${ }^{9,}, 10$ It would appear that social media and negative mood states are reciprocal at least in crosssectional studies like these depending on the somewhat arbitrary selection of independent and dependent variables. For example, those with higher anxiety levels in one study were noted to be internet addicted. ${ }^{11}$ In the same study, the authors selected age as an independent variable and internet use as the dependent measure and reported that younger age contributed to greater internet use. ${ }^{11}$ That finding was not surprising inasmuch as internet addiction has been particularly prevalent in younger individuals in many countries. ${ }^{10}$ In contrast, positive effects have also been noted for Internet use. For example, internet use was associated with less suicidal ideation in one study reputedly via less depression. ${ }^{12}$ The authors suggested that internet use may be reinforced by experiencing relief from negative feelings and that the positive effects were related to social relationship satisfaction that occurred on the Internet, especially for older adults.

Facebook time has been related to depression effects to such a degree that it has come to be called "Facebook depression" by some who have suggested that Facebook contributes to depression. ${ }^{13}$ Facebook users have not only been more depressed but have also experienced more social stress and insomnia even though, again, these were based on crosssectional data. ${ }^{14}$ Others have referred to excessive use as "Facebook intrusion" and have reported that being male and young as well as "spending more minutes online" have contributed to Facebook intrusion ${ }^{14,1}$ Still others have noted a greater prevalence in young males and referred to excessive use as "Facebooking"16

None of the above studies were conducted in times of social isolation like lockdowns. Based on these studies, social media might be expected to be even more related to negative emotions because of less activity and social contact during lockdowns like COVID-19. Alternatively, it is possible that social media could compensate for social isolation by providing social experiences and connections with others. In that way, social media might be expected to increase social connections and decrease stresses related to being socially isolated such as depression and anxiety. Although COVID-19 studies have assessed feelings of isolation and loneliness ${ }^{17}$, depression $^{18,} \quad{ }^{19}, \quad$ anxiety $^{20}$, sleep disturbances ${ }^{21,22}$, and PTSD symptoms ${ }^{23}$ only one of these COVID-19 lockdown studies referred to time on social media. ${ }^{17}$

The purpose of the present study was to explore the relationships between specific social media including those of texting, internet, and 
Facebook and physical and mental health during a COVID-19 lockdown. These included the relationships between social media, health and work activities as well as connecting with others and the earlier reported negative problems including stress, depression, anxiety, sleep disturbances and PTSD symptoms. Self-report data were collected via a survey on Survey Monkey and were analyzed via correlation analyses and ANOVAs.

\section{Methods}

\section{Participants}

A G* power analysis indicated that a sample size of 224 was required for an alpha of .05 and $80 \%$ power. The participants included individuals $(\mathrm{N}=260)$ who ranged in age from 18-82 ( $\mathrm{M}=47$ years). Gender was distributed $79 \%$ female, $18 \%$ male and $3 \%$ other (non-specified). Ethnicity was distributed 68\% Non-Hispanic White, 21\% Hispanic, 3\% Black and $8 \%$ other (non-specified). Professions were distributed $35 \%$ office worker, $30 \%$ academic, $15 \%$ managerial, $12 \%$ medical and $8 \%$ labor. The average income was $\$ 72,572,28 \%$ were unemployed and $69 \%$ worked at home. Twentythree per cent lived alone.

\section{Procedure}

A flyer was posted on Facebook giving a brief description of the study including some sample items and the age criterion being greater than 18 years. The Facebook flyer included a link to the survey on Survey Monkey which included 11 scales for a total of 87 items. The survey was four weeks duration (April 1-30, 2020), and the data were directly transported to SPSS for data analyses.

\section{Measures}

The survey included several demographic items including those already mentioned (age, gender, ethnicity, profession, income, type of employment, working at home, and living alone). The following five scales were created specifically for this survey to relate to activities and stress associated with the COVID-19 lockdown. ${ }^{24}$ The participants rated the items on the scales from zero meaning "not at all" to three meaning "a lot" including the:

1) Health Scale (15 items) (Cronbach's alpha=.66) which included exercise (inside exercise, outside exercise and outside exercise with others as well as the types of exercise), touching (touching partner, kids and self as well as the types of touching), COVID- 19-related safety practices including washing hands and social distancing, self-care, spiritual activities (meditating and feeling spiritual), and liking being at home. A factor analysis yielded three factors contributing to $47 \%$ of the variance on the Health Scale score: Factor 1 Self/Spiritual Care- Meditating (.74), Self-Care (.68), and Feeling Spiritual (.77)- $23 \%$ of the variance; Factor 2 Touching- Touching your kids (.75) and Touching your partner or friend (.72)- 14\% of the variance; Factor 3 Exercise- Outside exercise (-.89) and Exercise outside with someone else (-.76)- $10 \%$ of the variance.

2) Media/Communications Scale (10 items) (Cronbach's alpha $=.58$ ) including talking on the phone, texting, on Internet, gaming, on Facebook/Instagram, spending time receiving and sending messages/media about the virus, engaging in Zoom/Skype/Facetime activities (e.g. Yoga, meditation), watching the news, watching other TV programs, and watching movies. A factor analysis yielded four factors contributing to $61 \%$ of the variance on the Media/Communications Scale score: Factor 1 Entertainment-Watching movies (.84), TV programs (.80)- $23 \%$ of the variance; Factor 2 Communication- phone (.80), texting (.70). Zoom (.63)-14\% of the variance; Factor 3 Social Media- on internet (.78), Facebook (.60)$13 \%$ of the variance; and Factor 4-COVID News-watching the news (.79) and messaging about the virus (.60)- $11 \%$ of the variance;

3) Connecting Scale (4 items) (Cronbach's alpha $=.41$ ) which included connecting with friends, trying to connect with old friends, helping children do homework, and receiving support from others; 
4) Working Scale (6 items) (Cronbach's alpha=.61) including cooking, caregiving, housekeeping, paperwork, creative work, and working on projects/hobbies; and

5) Stress Scale (11 items) (Cronbach's alpha=.78) which included worrying about getting a virus, worrying about your financial status, wanting this experience to end, feeling isolated, feeling lonely, feeling bored, feeling touch deprived, snacking, drinking alcohol, napping, and getting "cabin fever". A factor analysis yielded three factors contributing to 56 $\%$ of the variance on the Stress Scale score: Factor 1 Stimulation deprivation- Feeling Isolated (.86), Feeling lonely (.86), Feeling bored (.74), Getting cabin fever (.70) and Feeling touch deprived (.65)- $34 \%$ of the variance; Factor 2 Worrying- Worried about finances (.67) and Worried about the virus (.47)- $12 \%$ of the variance; and Factor 3 Stress behaviorsNapping (.68) and Snacking (.53)- 10\% of the variance.

The standardized scales on the survey included 4 PROMIS Subscales ${ }^{25}$ (each item was rated on a 5 -point scale as $1=$ never, $2=$ rarely, $3=$ sometimes, $4=$ often, and $5=$ always) which included the : 1) PROMIS Anxiety Subscale (4 items) (Cronbach's alpha=.88) which included I felt fearful, I found it hard to focus on anything other than my anxiety, my worries overwhelmed me, and I felt uneasy;

2) PROMIS Depression Subscale (4 items) (Cronbach's alpha=.91) including I felt worthless, helpless, depressed, and hopeless;

3) PROMIS Fatigue Subscale (3 items) (Cronbach's alpha=.92) including I felt fatigued, I had trouble starting things because I'm tired, and I felt run-down; and

4) PROMIS Sleep Disturbance Subscale (4 items) (Cronbach's alpha $=.86$ ) which included my sleep quality was bad, my sleep is not refreshing, I had a problem with my sleep, and I had difficulty falling asleep.

The second standardized scale was a PTSD Screener entitled "PTSD-8: A short PTSD
Inventory" (8 items) (Cronbach's alpha $=.92){ }^{26}$ This inventory is introduced by the statement "If you're being reminded of a traumatic experience, please rate how much the following have bothered you during the lockdown" as: 0 ) not at all, 1) rarely, 2) sometimes, and 3) most of the time. The items are: recurrent thoughts and memories of the event, feeling as though the event is happening again, recurrent nightmares about the event, sudden emotional or physical reactions when reminded of the event, avoiding activities that remind you of the event, avoiding thoughts or feelings associated with the event, feeling jumpy/easily startled, and feeling on guard.

The last item on the COVID-19 Lockdown Activities survey was an open-ended question "Please tell us about anything you feel that has been positive about the lockdown." Survey Monkey then provided a listing of the most frequently used words and their percentiles for that item.

\section{Results}

\section{Correlation Analyses Yielding Significant Coefficients for Texting}

Results indicated that $98 \%$ of the sample reported texting (rated $0(2 \%)$ none, $1(16 \%), 2$ $(37 \%), 3(45 \%)$ a lot). Correlation analyses revealed a number of significant correlation coefficients for texting (at the $p<.05$ level with most at the $p=.000$ level) including a negative correlation between texting and living alone (.16 ), and the following (see table 1 for the correlation coefficients for the scales' total scores):1) a positive correlation for the Health Scale total score and for its items including more outdoor exercise with someone else, more selftouch, more washing hands and social distancing, and more self-care; 2) positive correlations for the Media/Communication Scale total score and its items indicating that texting was related to more phoning, more use of the internet, more use of Facebook, more use of Zoom and more watching tv programs ;3) a positive correlation with the total score on the Connecting Scale and its items connecting with IJPRR:https://escipub.com/international-journal-of-psychological-research-and-reviews/ 4 
friends and support from others; 4)a positive correlation with the Working Scale total score and the items including more housekeeping; and 5) a positive correlation with Stress Scale items including more worried about getting the virus and feeling touch deprived.

Table 1. Correlation coefficients for significant relationships between texting ratings and scores on COVID-19 Lockdown Activities Survey scales.

\begin{tabular}{lcc}
\hline Measure & Correlation coefficient & p level \\
\hline Health Scale Score & .23 & .000 \\
Media Scale Score & .50 & .000 \\
Connecting Scale Score & .34 & .000 \\
Working Scale Score & .17 & .005 \\
\hline
\end{tabular}

Table 2. Correlation coefficients for significant relationships between Internet ratings and scores on COVID-19 Lockdown Activities Survey scales and subscales.

\begin{tabular}{lcc}
\hline Measure & Correlation coefficient & p level \\
\hline Media Scale Score & .36 & .000 \\
Connecting Scale Score & .17 & .008 \\
Stress Scale Score & .15 & .01 \\
PROMIS Anxiety Subscale Score & .16 & .01 \\
PROMIS Depression Subscale Score & .15 & .02 \\
\hline
\end{tabular}

Correlation Analyses Yielding Significant Coefficients for Internet Use

Results indicated that $100 \%$ of the sample reported internet use (rated $0(0 \%)$ none, $1(3 \%)$, $2(120 \%), 3(77 \%)$ a lot). Correlation analyses revealed a number of significant correlation coefficients for internet use (at the $p<.05$ level) including a negative correlation between internet use and age and a positive correlation with having a routine and the following (see table 2 for the correlation coefficients for the scales' total scores):1) a negative correlation for Health Scale items including less outdoor exercise and less feeling spiritual; 2) a positive correlation on the Media/Communication Scale total score and its items including more texting, time on Facebook, more sending and receiving messages about the virus and more watching $\mathrm{tv} ; 3$ ) a positive correlation with the Connecting Scale total score and items suggesting more connecting with friends and more support from others; 4) a positive correlation with the Stress Scale total score and one item suggesting more cabin fever;5) a positive correlation with the PROMIS Anxiety Subscale score and all its items including feeling fearful, having difficulty focusing on anything other than my anxiety, worries overwhelming me and feeling uneasy; and 6) a positive correlation for the depression item on the PROMIS Depression Subscale total score and its items helpless and hopeless.

Correlation Analyses Yielding Significant Coefficients for Facebook Use Results indicated that $100 \%$ of the sample 
reported internet use (rated $0(0 \%)$ none, $1(3 \%)$, $2(20 \%), 3(77 \%)$ a lot). Correlation analyses revealed a number of significant correlation coefficients for Facebook use (at the $p<.05$ level) (see table 3 for the correlation coefficients for the scales' total scores): including:1) several demographic variables suggesting that Facebook was used more frequently by males and was negatively related to age, years of schooling, professional status, working at home during the lockdown, and routine but positively related to number of family members at home; 2 ) a negative correlation for the Health Scale item indicating less outside exercise and a positive correlation related to "touching your kids"; 3 ) positive correlations on the Media/Communication Scale total score and its items including more texting, more time on Internet, more sending and receiving messages about the virus, more watching tv, and more watching movies; 4) a positive correlation with the Connecting Scale item suggesting more time helping children with homework; 5) a positive correlation with the Stress Scale total score and several items suggesting feeling more isolated, lonely and bored and more snacking and napping ;6) a positive correlation with the PROMIS Anxiety Subscale score and all its items including feeling fearful, having difficulty focusing on anything other than my anxiety, worries overwhelming me and feeling uneasy; 7) a positive correlation for the PROMIS Depression Subscale total score and its items feeling helpless, depressed and hopeless; 8) a positive correlation for the PROMIS Fatigue Subscale total score and all its items including fatigue, tired, and rundown; 9) a positive correlation for the PROMIS Sleep Disturbance Subscale total score and all its items including sleep quality, refreshing sleep, problem sleeping, and difficulty falling asleep; and 10) a positive correlation for the Posttraumatic Stress Inventory-8 total score and its items including feeling that the traumatic experience is happening again, having nightmares, having emotional or physical reactions when reminded of the event, having thoughts or feelings associated with the event, and feeling jumpy/easily startled.

Table 3. Correlation coefficients for significant relationships between Facebook ratings and scores on COVID-19 Lockdown Activities Survey scales and subscales.

\begin{tabular}{lcc}
\hline Measure & Correlation coefficient & p level \\
\hline Media Scale Score & .53 & .000 \\
Stress Scale Score & .27 & .000 \\
PROMIS Anxiety Subscale Score & .25 & .000 \\
PROMIS Depression Subscale Score & .17 & .006 \\
PROMIS Fatigue Subscale Score & .16 & .009 \\
PROMIS Sleep Disturbance Subscale Score & .17 & .006 \\
Posttraumatic Stress-8 Inventory Score & .17 & .02 \\
\hline
\end{tabular}

Analyses of Variance (ANOVAs) on Texting group which was comprised of those Groups respondents with a 3 rating (a lot) on texting and ANOVAs were conducted to provide confirmatory data for the correlation analyses. The sample was divided into "a lot of texting" a "not a lot of texting" group that included participants who gave ratings of 0 (not at all) to 2 (moderate). These divisions were made to yield groups of approximately equal Ns. The ANOVAs 
comparing these groups provided confirmatory data for the scales and subscales that were significantly correlated with the texting variable including the Health, Media, Connecting and Working Scales (see table 4 for these ANOVAs). Exceptions to the ANOVAs providing confirmatory data for the correlation results included the following scale items: 1) ANOVAs were not significant for the Health scale item social distancing and for the Stress scale item feeling touch deprived; and 2) ANOVAs introduced new significant items including that more texting occurred at a younger age and those who texted a lot did more messaging about the virus (Media scale item) and engaged in more creative activities (Working scale item). The MANOVA for this set of ANOVAs was significant (Wilks' Lambda $F=15.36, p=.000$, eta squared $=.23$ ).

Table 4. Mean scale scores for significant ANOVAs for a" lot of texting" (3 rating) group versus a "no texting, a little and moderate texting" (0-2) group (standard deviations in parentheses).

\begin{tabular}{llllll}
\hline Measure & 0-2 group & 3 group & $\mathrm{F}$ & $\mathrm{p}$ & eta $^{2}$ \\
\hline Health & $31.00(5.55)$ & $32.71(5.31)$ & 5.44 & .02 & .02 \\
Media & $24.05(3.89)$ & $27.63(4.03)$ & 34.98 & .000 & .12 \\
Connecting & $8.17(2.03$ & $9.04(2.33)$ & 6.23 & .01 & .03 \\
Working & $25.31(5.63)$ & $19.69(3.03)$ & 124.14 & .000 & .33 \\
\hline
\end{tabular}

Table 5. Mean scale scores for significant ANOVAs for a" lot of Internet" (3 rating) group versus a "no Internet, a little and moderate Internet" (0-2) group (standard deviations in parentheses).

\begin{tabular}{lccrcc}
\hline Measure & 0-2 group & \multicolumn{1}{c}{3 group } & $\mathrm{F}$ & $\mathrm{p}$ & eta $^{2}$ \\
\hline Media & $24.05(3.89)$ & $27.63(4.03)$ & 34.98 & .000 & .12 \\
Connecting & $8.17(2.03$ & $9.04(2.33)$ & 6.23 & .01 & .03 \\
Stress & $25.31(5.63)$ & $19.69(3.03)$ & 124.14 & .000 & .33 \\
Anxiety & $8.68(3.31)$ & $10.22(3.56)$ & 6.55 & .01 & .03 \\
Depression & $7.19(3.09)$ & $8.70(4.08)$ & 6.68 & .01 & .03 \\
\hline
\end{tabular}

Analyses of Variance (ANOVAs) on Internet Groups

ANOVAs were conducted to provide confirmatory data for the correlation analyses. The sample was divided into a group of those respondents with a 3 rating (a lot) on internet use and a group of participants who gave ratings of 0 (not at all) to 2(moderate) for internet use. These divisions were made to yield groups of approximately equal Ns. The ANOVAs comparing these groups provided confirmatory data for the scales and subscales that were significantly correlated with the texting variable. These included the Connecting, Media and Stress scales and the Anxiety and Depression subscales (see table 5 for the Internet group effects on the survey scales and subscales). The ANOVAs also yielded significant internet effects for additional items including the Stress Scale items feeling lonely, snacking and drinking IJPRR:https://escipub.com/international-journal-of-psychological-research-and-reviews/ 7 
alcohol and the Depression item feeling worthless. The MANOVA for this set of ANOVAs was significant (Wilks' Lambda $F=7.16, p=.000$, eta squared $=.14$ ).

\section{Analyses of Variance (ANOVAs) on Facebook Groups}

ANOVAs were conducted to provide confirmatory data for the correlation analyses. The sample was divided into a group of those respondents with a 3 rating (a lot) on Facebook use and a group of participants who gave ratings of 0 (not at all) to 2(moderate) for Facebook use. These divisions were made to yield groups of approximately equal Ns. The ANOVAs comparing these groups provided confirmatory scales and subscales that were significantly correlated with the texting variable. These included the Media and Stress scales and the Anxiety subscales (see table 6 for the Facebook group effects on the survey scales and subscales). However, the Depression, Fatigue and Sleep Subscales and the PTSD scale that were significantly correlated with Facebook use did not significantly differentiate the Facebook groups. Similarly, some correlated items were not significant Facebook effects indicating less outside exercise (Health scale item), more napping (Stress scale item), and more feeling uneasy (Anxiety Subscale item). The ANOVAs also yielded significant group differences on the following items that were not significantly correlated with Facebook use indicating less feeling spiritual (Health scale item), less connecting with friends (Connecting scale item), and wanting this experience to end, feeling touch deprived and getting "cabin fever" (Stress scale items). The MANOVA for this set of ANOVAs was significant (Wilks' Lambda $F=34.99, p=.000$, eta squared $=.30$ ).

Table 6. Mean scale scores for significant ANOVAs for a" lot of Facebook" (3 rating) group versus a "no Facebook, a little and moderate Facebook" (0-2) group (standard deviations in parentheses).

\begin{tabular}{lllccc}
\hline Measure & $0-2$ group & 3 group & $F$ & $p$ & eta $^{2}$ \\
\hline Media & $24.90(3.75)$ & $29.53(3.39)$ & 101.52 & .000 & .29 \\
Stress & $25.90(5.93)$ & $28.95(6.43)$ & 15.24 & .000 & .06 \\
Anxiety & $9.43(3.62)$ & $10.44(3.46)$ & 4.93 & .03 & .02 \\
\hline
\end{tabular}

\section{Discussion}

Social media had both positive and negative relationships to physical and mental health in this COVID-19 lockdown survey sample. Texting had primarily positive effects while those for Internet use were mixed (both positive and negative effects) and those for Facebook time were solely negative. That the effects differed across these three forms of social media was surprising, although they have not been compared in pre-COVID research and they have not been assessed by COVID-19 researchers.

Texting was, as might be expected, related to other social media activities including phoning, internet, Facebook, Zoom and watching tv. The positive relationships for texting included engaging in more health-related activities like exercising with someone else, self-touch, and self-care, as well as connecting with friends, and engaging in more housekeeping. These data were inconsistent with pre-COVID literature which suggested that texting was only associated with negative problems including greater anxiety, ${ }^{3}$ depression, ${ }^{4,5}$ and sleep disturbances. ${ }^{6}$ These inconsistent data may relate to the previous literature being based on excessive use of texting, ${ }^{5,6}$ while only $45 \%$ of this COVID-19 sample claimed to be texting "a lot". 
The only negative relationships for texting in this sample were "worrying about the virus" and "feeling touch deprived". Greater worry about the virus may relate to text messaging more about the virus. Feeling touch deprived was surprisingly unique to this form of social media in this sample. But greater texting has appeared to be associated with less touch even in nonCOVID samples such as an airport gate observation study that is suggesting excessive time on cell phones and virtually no time touching travel partners. ${ }^{27}$

Internet use was also not surprisingly related to other forms of social media including texting, Facebook time, watching TV, and communicating about the virus. Both positive and negative relationships were noted for internet use. The positive included connecting with others and feeling supported. These were unique to the internet literature, likely because they have not been assessed in previous research. The use of internet by younger adults was consistent with previous literature suggesting negative associations between internet use and age. ${ }^{10,11}$

More negative than positive relationships were noted for internet use in this COVID-19 sample including greater stress, anxiety, and depression. In addition, those who spent more time on internet did not have a routine, engaged in less outdoor exercise, and did not feel spiritual which could have contributed to their stress. Exercise has alleviated touch deprivation, and feeling spiritual has related to less stress during COVID-19 lockdowns. ${ }^{24,28}$ The associations between Internet use and depression and anxiety are consistent with pre-COVID literature, ${ }^{8-10}$ and even with research on Internet addiction. ${ }^{10,11}$ Although this sample was not assessed for Internet addiction, as many as 77\% reported using Internet "a lot". Unfortunately, "a lot" does not accurately quantify time on social media.

Facebook time was reported by $91 \%$ this sample, with $42 \%$ rating time on Facebook as "a lot". This prevalence is perhaps not surprising inasmuch as the flyer for this Survey Monkey study was posted on Facebook. Not unlike texting and internet use, Facebook time was related to other social media including texting, Internet use, messaging about the virus, and watching TV and movies. That more users were young and male is consistent with previous data. ${ }^{14,16}$ Other contributing demographic factors were having less schooling, having less professional work, not working currently at home, and having no routine, all of which may have confounded the effects of Facebook time.

Facebook time was not only associated with stress and anxiety but was also significantly correlated with depression, fatigue, sleep disturbances and PTSD symptoms. These data are consistent with the social stress and insomnia reported by some ${ }^{14}$ and the depression called "Facebook depression", ${ }^{13}$ and "Facebook intrusion" 15 noted by others. "Facebooking" individuals were also feeling less spiritual, feeling more touch deprived, and engaging in less outdoor exercise which would be expected to exacerbate their stress inasmuch as feeling spiritual and engaging in more outdoor exercise have been noted to alleviate the stressful effects of COVID- 19 lockdowns. ${ }^{24,28}$ Facebook time was also correlated with some items that were considered healthy including connecting with friends, family members living at home, doing homework with kids, and touching kids. But, unfortunately these activities did not buffer the negative effects of Facebook time.

These data are inconclusive due to limited sampling and assessment issues. The recruitment via Facebook may have contributed to its being a predominantly non-Hispanic, white female sample. This would be considered a nonrepresentative sample even though it may be a representative Survey Monkey sample. The selfreport data, as always, are prone to potential reliability problems and bias effects. And, causality or direction of effects cannot be determined from this cross-sectional survey. Longitudinal studies are needed to provide baseline and follow-up data. In addition, daily 
diaries or recording technology would provide more reliable measures of texting, internet use, and Facebook time.

Nonetheless, these data suggest differential effects of different types of social media during lockdowns that appear to be on a continuum from positive effects for texting to both positive and negative effects for internet use to negative effects for Facebook time. The negative effects of "a lot" of internet and Facebook time including anxiety and depression symptoms are consistent with pre-COVID literature. These negative effects may have been exacerbated by the lack of routine and outdoor exercise which were uniquely assessed in this COVID-19 sample. These survey data also revealed positive, novel effects of texting on health and connecting activities and internet use for connecting with friends. Further research is needed on texting, internet and Facebook effects as they will likely continue being prevalent COVID-19 activities.

\section{References}

1. Rathbone, A.L. \& Prescott, J. The use of mobile apps and SMS messaging as physical and mental health interventions: Systematic review. Journal of Medical Internet Research, 2017, 19(8):e295. Doi:10.2196/jmir.7740.

2. Kelders, S.M., Bohlmeijer, E.T., Pots, W.T.M. \& van Gemert-Pijnen, J.E.W.C. Comparing human and automated support for depression: Fractional factorial randomized controlled trial.Behavior Research and Therapies, 2015;72:7280. Doi:10.1016/j.brat.2015.06.014. Epub 2015 Jul 6.

3. Visnjic, A., Velickovic, V., Sokolovic, D., Stankovic, M., Mijatovic, K., Stojanovic, M. et al. Relationship between the manner of mobile phone use and depression, anxiety, and stress in university students. International Journal of Environmental Research and Public Health, 2018 15(4):697. Doi: 10.3390/ijerph15040697.

4. Kim, Y.J., Jang, H.M., Lee, Y., Lee, D. \& Kim, D.J. Effects of internet and smartphone addictions on depression and anxiety-based propensity score matching analysis. International Journal of Environmental Research and Public Health, 2018 15(5):859. Doi:10.3390/ijerph15050859.

5. Field, T. Cell phone addiction in adolescents: A narrative review. 2018. Journal of Addiction and
Adolescent Behavior. 3 (4), 12-22. DOI 10.3352/OAJAP.2020.03.00566.

6. Thomee, S. Mobile phone use and mental health. A review of the research that takes a psychological perspective on exposure. International Journal of Research and Public Health, $2018 \quad$ 15(12):2692. Doi: 10.3390/ijerph15122692.

7. Bhat, S., Pinto-Zipp, G., Upadhyay, H. \& Polos, P.G. "To sleep perchance to tweet": In-bed electronic social media use and its association with insomnia, daytime sleepiness, mood, and sleep duration in adults. Sleep Health, 2018 Apr;4(2):166-173.

Doi: 10.10.16/j.sleh.2017.12.004. Epub 2018 Jan 17.

8. McDougall, M.A., Walsh, M., Wattier, K., Knigge, R., Miller, L., Stevermer, M. et al. The effect of social networking sites on the relationship between perceived social support and depression. Psychiatry Research, 2016 Dec 30;246:223-229.

Doi:

10.1016/j.psychres.2016.09.018. Epub 2016 Sep 14.

9. Kim, Y.J., Jang, H.M., Lee, Y., Lee, D. \& Kim, D.J. Effects of internet and smartphone addictions on depression and anxiety-based propensity score matching analysis. International Journal of Environmental Research and Public Health, 2018 15(5):859. Doi:10.3390/ijerph15050859.

10. Field, T. Internet addiction in adolescents: A narrative review. 2019. Journal of Addictions and Therapies 1, 1-11.

11. Soulioti, E., Stavropoulos, V., Christidi, S., Papastefanou, Y. \& Roussos, P. The relationship of internet addiction with anxiety and depressive symptomology. Psychiatriki, Apr-Jun 2018;29(2):160-171. Doi: 10.22365/jpsych.2018.292.160.

12. Jun, H.J. \& Kim, M.Y. What accounts for the relationship between internet use and suicidal ideation of Korean older adults? A mediation analysis. Journal of Geronatology. Series B. Psychology sciences and Social Sciences, 2017v;72(5):846-855. 10.1093/geronb/gbw163.

13. Yoon, S., Kleinman, M., Mertz, J. \& Brannick, M. Is social network site usage related to depression? A meta-analysis of Facebookdepression relations. Journal of Affective Disorders, 2019; 248:65-72. Doi: 10.1016/j.jad.2019.01.026. Epub 2019 Jan 27.

14. Brailovskaia, J., Margraf, J., Schillack, H. \& Kollner, V. Comparing mental health of Facebook users and Facebook non-users in an inpatient sample in Germany. Journal of Affective 
Disorders, 2019 Dec 1; 259:376-381. Doi: 10.1016/j.jad.2019..08.078.Epub 2019 Aug 24.

15. Blachnio, A., Przepiorka, A. \& Pantic, I. Internet use, Facebook intrusion, and depression: Results of a cross-sectional study. European Psychiatry, 2015 Sep;30(6):681-684. Doi: 10.1016/j.eurpsy.2015.04.002. Epub 2015.

16. Field, T. Facebooking in adolescents: A narrative review. 2019. Journal of Addiction and Adolescent

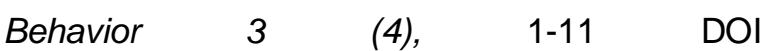
10.33552/OAJAP.2020.03.600567.

17. Field, T., Poling, S. Mines, S., Bendell, D. \& Veazey, C. Feeling isolated and lonely during a COVID-19 lockdown. 2020. Archives of Health Science, 4 (1), 1-9. DOI: 10:31829/2641:7456/ahs 2020-4 (1)-121.

18. Stanton, R., To, Q., Khalesi, S., Williams, S., Alley, S., Thwaite, T., Fenning, A. \& Vandelanotte, C. Depression, Anxiety and Stress during COVID-19: Associations with Changes in Physical Activity, Sleep, Tobacco and Alcohol Use in Australian Adults International Journal of Environmental Research and Public Health. 2020; 17(4065):4065 DOI 10.3390/ijerph17114065

19. Field, T., Mines, S., Poling, S., Bendell, D. \& Veazey, C. Anxiety and depression during a COVID-19 lockdown.2020. Journal of Anxiety and Depression, 3 (2), 124-137.

20. Huang, Y. \& Zhao, N. Generalized anxiety disorder, depressive symptoms and sleep quality during COVID-19 outbreak in China: A web-based cross-sectional survey, 2020, Psychiatry Research, 288, 112954.

21. Cellini, N., Canale, N. Mioni, G., \& Costa, S. Changes in sleep pattern, sense of time and digital media use during COVID-19 lockdown in Italy, Journal of Sleep Research 2020, doi. org/10.1111/jsr.13074

22. Field, T., Mines, S., Poling, S., Bendell, D. \& Veazey, C. Stress and sleep disturbances during a COVID-19 lockdown. 2020 Psychology and Mental Health, 4, 1-5. DOI: 10:31579/26378892/092.

23. Mines, S., Veazey C., Poling, S., Field, T. \& Bendell, D. PTSD symptoms and health behaviors. 2020. Annals of Psychiatry and Mental Health. 8(3), 1155-1159.

24. Field, T., Poling, S., Mines, S., Bendell, D. \& Veazey, C. Touch deprivation and exercise during a COVID-19 lockdown.2020. Medical Research Archives, 8 (8), 1-12.

25. Dewitt, B., Feeny, D., Fischhoff, B., Celia, D., Hays, R.D. et al. Estimation of a preferencebased summary score for the patient reported outcomes measurement information system: The
PROMIS-preference (PROPr) scoring system. Medical Decision Making. 2018: 38: 683-698.

26. Hansen, M., Anderson, T. E., Armour, C., Elklit, A., Palic, S., \& Mackrill, T. PTSD-8: A short PTSD inventory. Clinical Practice \& Epidemiology in Mental Health. 2010:6:101- 108.

27. Field, T., Mines, S. \& Poling, S. Cell phones dominate waiting activity at airport gates. 2020 International Journal of Psychology Research and Reviews. 4:47-52.

28. Field, T., Poling, S. , Mines, S., Bendell, D. \& Veazey, C. Spirituality and meditation during a COVID-19 lockdown. 2020. In review. 